\title{
A!
}

This is an electronic reprint of the original article.

This reprint may differ from the original in pagination and typographic detail.

Bagheri, Mohammad; Ibragimova, Rina; Komsa, Hannu Pekka

\section{Fermiology of two-dimensional titanium carbide and nitride MXenes}

Published in:

Physical Review B

DOI:

10.1103/PhysRevB.104.035408

Published: 07/07/2021

Document Version

Publisher's PDF, also known as Version of record

Please cite the original version:

Bagheri, M., Ibragimova, R., \& Komsa, H. P. (2021). Fermiology of two-dimensional titanium carbide and nitride MXenes. Physical Review B, 104(3), [035408]. https://doi.org/10.1103/PhysRevB.104.035408

This material is protected by copyright and other intellectual property rights, and duplication or sale of all or part of any of the repository collections is not permitted, except that material may be duplicated by you for your research use or educational purposes in electronic or print form. You must obtain permission for any other use. Electronic or print copies may not be offered, whether for sale or otherwise to anyone who is not an authorised user. 


\title{
Fermiology of two-dimensional titanium carbide and nitride MXenes
}

\author{
Mohammad Bagheri $\odot,{ }^{1}$ Rina Ibragimova $\odot,{ }^{2}$ and Hannu-Pekka Komsa $\oplus^{1,2}$ \\ ${ }^{1}$ Microelectronics Research Unit, University of Oulu, 90014 Oulu, Finland \\ ${ }^{2}$ Department of Applied Physics, Aalto University, 00076 Aalto, Finland.
}

(Received 22 April 2021; revised 22 June 2021; accepted 23 June 2021; published 7 July 2021)

\begin{abstract}
MXenes are a family two-dimensional transition-metal carbide and nitride materials, which often exhibit very good metallic conductivity and are thus of great interest for applications in, e.g., flexible electronics, electrocatalysis, and electromagnetic interference shielding. However, surprisingly little is known about the fermiology of MXenes, i.e., the shape and size of their Fermi surfaces, and its effect on the material properties. One reason for this may be that MXene surfaces are almost always covered by a mixture of functional groups, and studying Fermi surfaces of disordered systems is cumbersome. Here, we study fermiology of four common Ti-based MXenes as a function of the surface functional group composition. We first calculate the effective band structures of systems with explicit mixed surfaces and observe gradual evolution in the filling of the Ti-d band and resulting shift of Fermi level. We then demonstrate that these band structures can be closely approximated by using pseudohydrogenated surfaces, and also compare favorably to the experimental angle-resolved photoemission spectroscopy results. By modifying the pseudohydrogen charge we then proceed to plot Fermi surfaces for all systems and extract their properties, such as the Fermi-surface area and average Fermi velocity. These are in turn used to evaluate the electrical conductivity with the relaxation time fitted to experimentally measured conductivities.
\end{abstract}

DOI: 10.1103/PhysRevB.104.035408

\section{INTRODUCTION}

MXenes are a large class of two-dimensional (2D) materials of transition-metal $(\mathrm{M})$ carbides and nitrides $(\mathrm{X})[1,2]$. Distinct from other 2D materials, MXenes are synthesized by etching out layers of atoms from a layered bulk precursor phase. As a result of the etching process, the dangling bonds on the surface are passivated by a mixture of organic groups from the etching solution, such as $-\mathrm{O},-\mathrm{OH}$, and $-\mathrm{F}$ [3]. MXenes are metallic and exhibit very high conductivity in addition to good mechanical properties and easy solution processing. As a result, they have shown great promise for applications in conductive inks, battery electrodes, electromagnetic interference shielding, and various types of sensors [4-7].

Given the large interest on these materials, surprisingly little effort has been devoted to understanding the fundamental properties of their Fermi surfaces, i.e., fermiology, and its effect on the material properties [8]. Experimentally, Fermi surfaces are commonly investigated using angle-resolved photoemission spectroscopy (ARPES) [8,9]. ARPES results for delaminated $\mathrm{Ti}_{3} \mathrm{C}_{2}$, the prototypical MXene, were reported by Schultz et al. [10], but since the measured film consists of randomly oriented flakes, the ARPES data was azimuthally averaged, which prevented the extraction of the Fermi-surface shape. Quasiparticle interference within scanning tunneling microscope (STM-QPI) is also often used to gain insight on the Fermi surface, but requires high-quality surface with small density of scattering centers [11] - a luxury not afforded in the case of MXenes, where the surfaces are covered by a random mixture of organic groups and other residues from the synthesis. In fact, to the best of our knowledge, clean
STM images of MXene surfaces have not been reported in the literature. Finally, Fermi surfaces could be probed via de Haas van Alphen effect [12], but again no reports exist for MXenes.

Also computational studies of Fermi surfaces are very rare, even when the band structures have been reported in numerous papers and are relatively well understood [13-15]. Hu et al. calculated the Fermi surface of monolayer and bulk $\mathrm{Ti}_{2} \mathrm{C}_{2}(\mathrm{OH})_{2}$ for two different layer stackings [16]. In addition, some insight can be gained from the Fermi surfaces of the precursor MAX phases, which were presented in Ref. [12]. While the Fermi surfaces for pure $\mathrm{O}$ - or $\mathrm{OH}$-terminated structures are straightforward to evaluate, since the surfaces are passivated by a mixture of groups (stabilized by strong nearest neighbor interactions between the groups $[17,18]$ ) and the electronic structure of $\mathrm{O}$ - and $\mathrm{OH}$-terminated surfaces differ markedly, it is not clear how the Fermi surface evolves with changing functional group composition. Calculations for the mixed group surfaces are cumbersome due to requiring the use of supercells and the resulting band folding. The band structures can be unfolded with the effective band structure method, where each state from the supercell Brillouin zone is projected to each of the folded plane waves from the primitive cell Brillouin zone [19-22], but smearing of the bands still complicates the analysis.

In this paper, we show how to overcome these problems and report results for the Fermi surfaces of $\mathrm{Ti}_{3} \mathrm{C}_{2}, \mathrm{Ti}_{2} \mathrm{C}$, $\mathrm{Ti}_{4} \mathrm{~N}_{3}$, and $\mathrm{Ti}_{2} \mathrm{~N}$ monolayers with varying $\mathrm{O} / \mathrm{OH}$ composition. We calculate the effective band structures for mixed group surfaces and show that they can be well approximated by pseudohydrogenated surfaces. We analyze the 
(a)

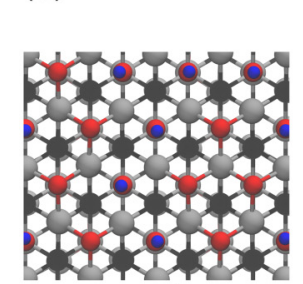

top view

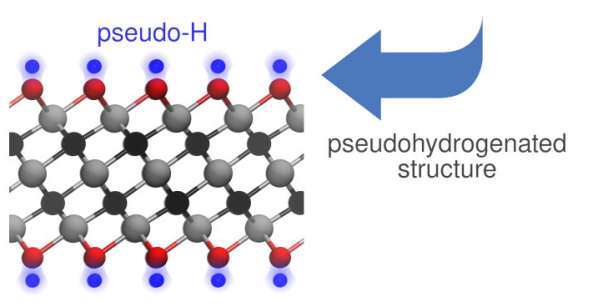

(b)

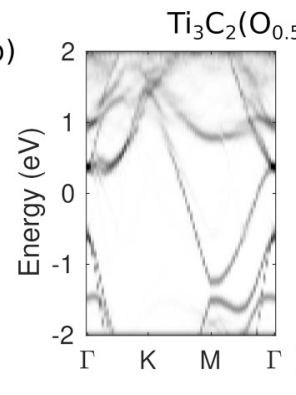

(c)

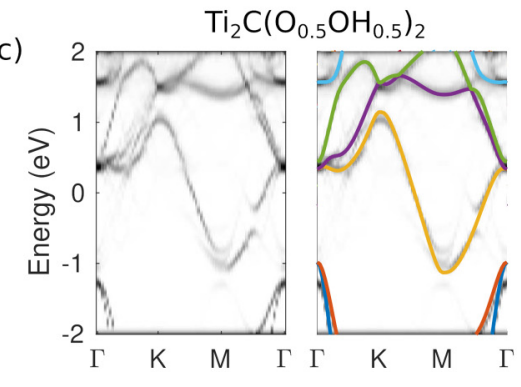

(d)

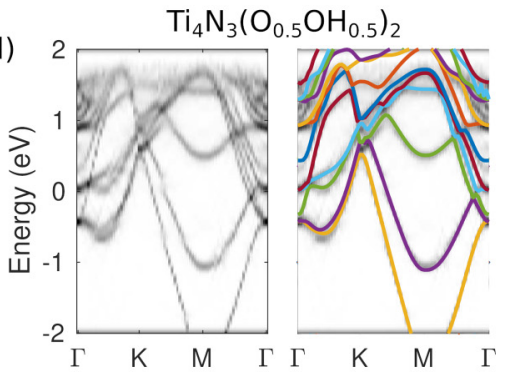

(e)

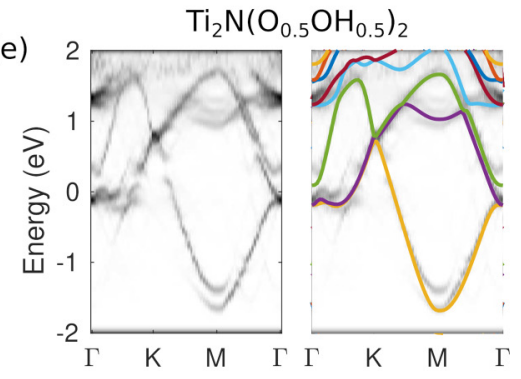

FIG. 1. (a) Atomic structure for $\mathrm{Ti}_{3} \mathrm{C}_{2}$ with a surface functionalized by a mixture of $\mathrm{O}$ and $\mathrm{OH}$ groups (top) and the corresponding pseudohydrogenated structure (bottom). (b)-(e) Effective band structures for all materials (left panel) and band structures of pseudohydrogenated surfaces overlaid on the EBS (right panel). Fermi level is set to zero.

Fermi-surface properties as a function of the surface group composition and compare the evaluated electrical conductivities to the experiments. We also compare the $\mathrm{Ti}_{3} \mathrm{C}_{2}$ band structure to those obtained from recent ARPES experiments.

\section{METHODS}

All density-functional theory calculations are carried out using VASP software [23,24], together with projector augmented plane wave method [25]. We adopt the PerdewBurke-Ernzerhof exchange-correlation functional for solids (PBEsol) [26] based on the benchmarking carried out in Ref. [17]. The plane wave cutoff is $550 \mathrm{eV}$ and the Brillouin zone of the primitive cell is sampled with a $16 \times 16 k$-point mesh.

The effective band structures (EBS) are calculated using BANDUP software [27]. The atomic structures for the mixed surfaces were determined via combination of cluster expansion and Monte Carlo simulations in our previous publication [18], and we use the same $4 \times 4$ supercell special quasiordered structures as constructed therein.

Electronic band structures are interpolated using BOLTZTRAP2 software, which is also used for the integration of the transport properties [28]. We use $k$-point mesh interpolation factor 200 and temperature $300 \mathrm{~K}$. Fermi surfaces are analyzed and plotted using the IFERMI software [29], with interpolation factor 20 .

\section{RESULTS}

\section{A. Band structures of mixed surface MXenes}

More realistic structural models for the distribution of functional groups were developed by us in Refs. $[17,18]$. As an example, the structure of $\mathrm{Ti}_{3} \mathrm{C}_{2}$ with $\mathrm{O}_{0.5} \mathrm{OH}_{0.5}$ surface composition is shown in Fig. 1(a). In the left panels of Figs. 1(b)-1(e) we show the effective band structures for the four Ti-based MXenes with $\mathrm{O}_{0.5} \mathrm{OH}_{0.5}$ surface composition. From these results, and from comparison to the band structure of pure surfaces (see Figs. 2-3 below), it is clear that the band structures undergo a gradual evolution and there are no extraneous bands arising from the surface groups. The band(s) at the Fermi level arise from the nonbonding $t_{2 g}$ states of Ti-d character [30]. Consequently, it appears that the main effect of $\mathrm{H}$ atoms is to control the electron concentration and consequently the Fermi-level position in the metal d bands, as has been previously assumed [14,15]. This is supported by the fact that the EBS is fairly insensitive to the arrangement of the functional groups, as shown in Fig. S2 [31], and suggests that also other charge acceptor or donor species could be used for controlling the Fermi-level position.

Given that effective band structures are unwieldy for detailed studies of these materials' fermiology, we propose to use pseudohydrogenated surfaces to control the Fermilevel position. In practice, we use the primitive cell of a fully $\mathrm{OH}$-terminated structure, but with the $\mathrm{H}$ atoms replaced by a pseudohydrogen atoms with atomic number $Z=0.25$, 0.50 , and 0.75 (i.e., proton charge is $Z e$ and total electron charge $-\mathrm{Ze}$ ) to model surface compositions of $\mathrm{O}_{0.75} \mathrm{OH}_{0.25}$, $\mathrm{O}_{0.5} \mathrm{OH}_{0.5}$, and $\mathrm{O}_{0.25} \mathrm{OH}_{0.75}$, respectively. The pseudohydrogen band structures for $Z=0.5$ are shown on the right panels of Figs. 1(b)-1(e), overlaid on top of the effective band structures. In all cases, the band structures of pseudohydrogenated MXenes agree extremely well with the EBS, thereby further corroborating the physical picture described above.

It is worth noting that we also calculated the band structures for systems with one side fully terminated by $\mathrm{O}$ and the other by $\mathrm{OH}$, but the resulting band structures did not agree favorably with the EBS due to broken symmetry. Moreover, such approach would obviously be limited to a $\mathrm{O}_{0.5} \mathrm{OH}_{0.5}$ composition.

The band structure evolution with $\mathrm{O} / \mathrm{OH}$ composition, for all materials and five different $\mathrm{O} / \mathrm{OH}$ compositions, are 

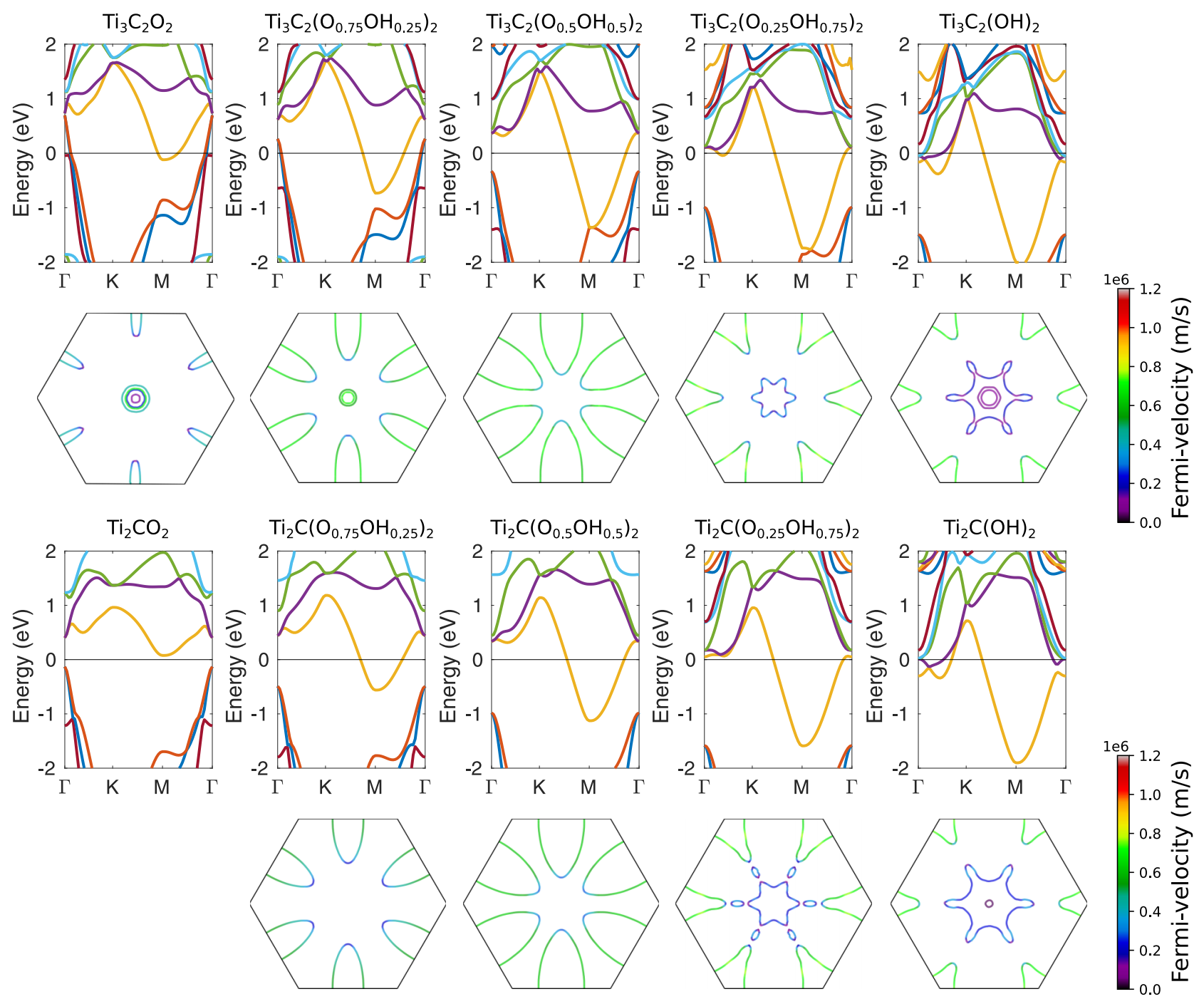

FIG. 2. Evolution of the band structures of $\mathrm{Ti}_{3} \mathrm{C}_{2}$ and $\mathrm{Ti}_{2} \mathrm{C}$ with surface group composition. The Fermi surfaces are plotted under each panel, with the color denoting Fermi velocity.

collected in Figs. 2 and 3. From these plots, the gradual change in the band energies is easily resolved.

Based on the analysis by $\mathrm{Hu}$ et al. [30], the states below Fermi level (red colored band and below in Figs. 2 and 3) arise from bonding state between Ti-d and C-p/N-p $\left(\mathrm{e}_{\mathrm{g}}\right)$, and similar to bulk carbides and nitrides [32]. The states at and above Fermi level (orange colored band and above) arise from nonbonding $\mathrm{t}_{2 \mathrm{~g}}$ states of Ti-d. In most cases there is a gap between these two band manifolds, but the Fermi level resides in the Ti-d manifold in all cases except for the fully O-terminated $\mathrm{Ti}_{2} \mathrm{C}$. Due to the larger electronegativity of $\mathrm{N}$, as compared to $\mathrm{C}$, the Ti-N bonding states are clearly lower in energy and lead to a larger gap below the nonbonding states. The Ti-C bands cross the Fermi level around $\Gamma$ point only for O-rich $\mathrm{Ti}_{3} \mathrm{C}_{2}$, but thereby prevent it from becoming semiconductor as happens with $\mathrm{Ti}_{2} \mathrm{CO}_{2}$.

Due to the additional electron(s) in N, as compared to C, there is one additional electron per $\mathrm{X}$ atom to fill the $\mathrm{M}-\mathrm{d}$ bands and consequently Fermi level resides higher within this band. For instance, the lowest-energy band (orange) is exactly half-filled by the one additional electron in the case of $\mathrm{Ti}_{2} \mathrm{~N}$, whereas the band is empty in the case of $\mathrm{Ti}_{2} \mathrm{C}$. Similar halffilling of the band can be obtained in the case of $\mathrm{O}_{0.5} \mathrm{OH}_{0.5}$ surface composition of $\mathrm{Ti}_{3} \mathrm{C}_{2}$ or $\mathrm{Ti}_{2} \mathrm{C}$.

The band fillings can also be readily obtained via electron counting. In the case of $\mathrm{Ti}_{3} \mathrm{C}_{2} \mathrm{O}_{2}$ and $\mathrm{Ti}_{2} \mathrm{CO}_{2}, \mathrm{Ti}, \mathrm{C}$, and $\mathrm{O}$ are in the oxidation states $+4,-4$, and -2 , respectively, as in $\mathrm{TiC}$ and $\mathrm{TiO}_{2}$. Upon addition of $\mathrm{H}$ atoms (oxidation state +1 ) and the subsequent occupation of the nonbonding Ti-d band, the charge balance is achieved by decreasing the oxidation state of Ti. The nitrogen atoms (oxidation state -3 ) have a similar effect.

$\mathrm{O} / \mathrm{OH}$ composition controls the occupation of the $\mathrm{t}_{2 \mathrm{~g}}$ band, but since it is nonbonding, it should have a relatively minor effect on the energetics. Among the purely terminated surfaces, O-terminated surfaces have been predicted to be the most stable, but the mixed surfaces arise in practice due to the strong attractive interactions between the opposite type surface groups $[17,18]$. Consequently, since the surface composition is dominated by the interactions between surface groups, it is rather independent on the $\mathrm{M}$ and $\mathrm{X}$ species, as we observed in Ref. [18] (we also note, that the energy gain 

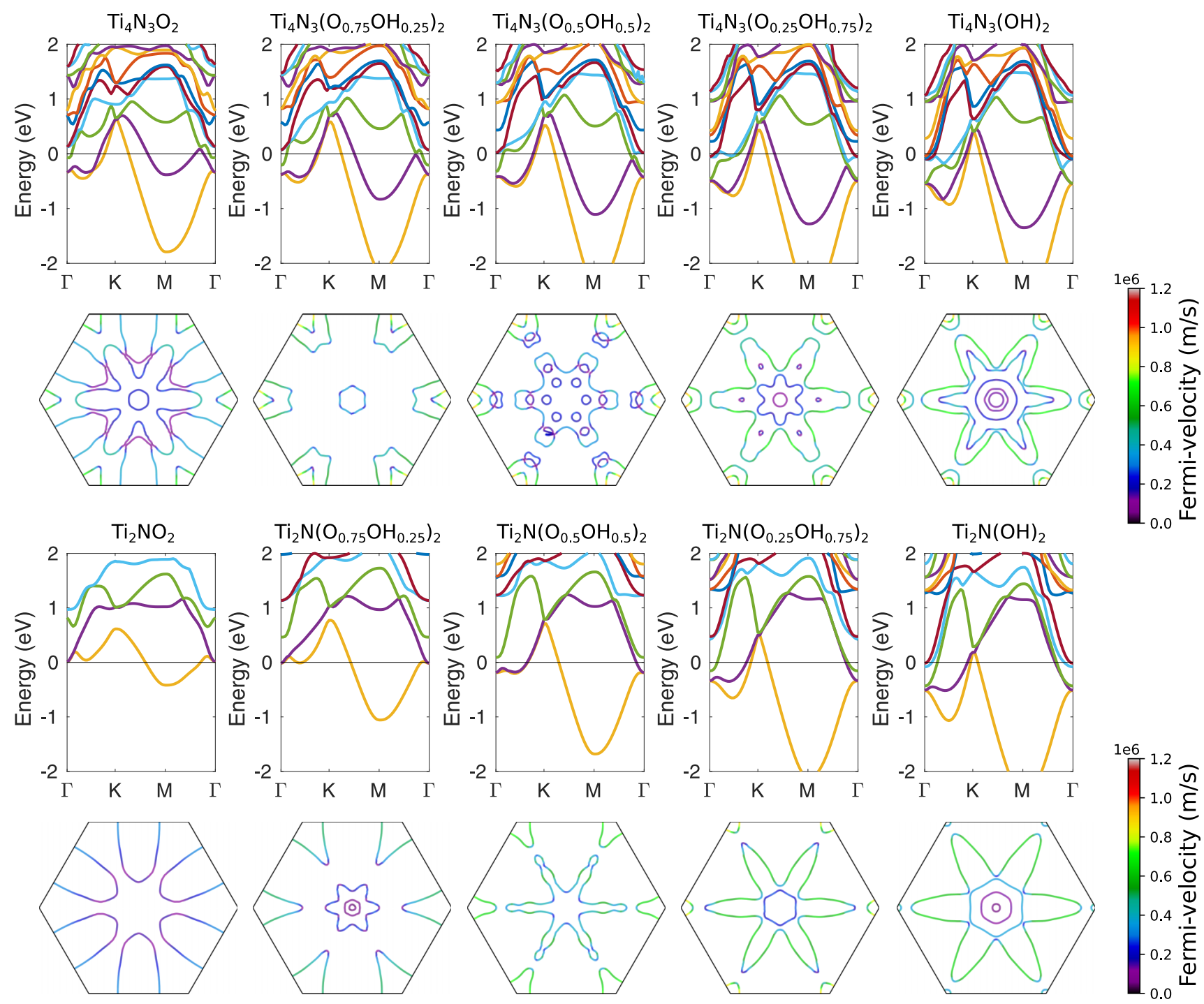

FIG. 3. Evolution of the band structures of $\mathrm{Ti}_{4} \mathrm{~N}_{3}$ and $\mathrm{Ti}_{2} \mathrm{~N}$ with surface group composition. The Fermi surfaces are plotted under each panel, with the color denoting Fermi velocity.

upon mixing is not captured in the pseudohydrogen models). Even if the $\mathrm{O} / \mathrm{OH}$ mixing cannot be easily avoided, we expect that the Fermi-level position can still be tuned by introducing alloying in the $\mathrm{M}$ and $\mathrm{X}$ sublattices.

\section{B. Fermi-surface properties}

The Fermi surfaces are plotted below the band structures in Figs. 2 and 3, and colored by the Fermi velocity. When Fermi level crosses the lowest-energy Ti-d band, it leads to hexagonally symmetric ellipsoids around the $\mathrm{M}$ points. These have fairly large Fermi velocities, reaching values above $0.5 \times 10^{6}$ $\mathrm{m} / \mathrm{s}$, which is only about factor of two lower than in the highly conducting elemental metals. At large $\mathrm{OH}$ concentrations (or any concentration in the case of $\mathrm{Ti}_{4} \mathrm{~N}_{3}$ ) when the Fermi level rises to cross the set of states around $\Gamma$ point, the Fermi surfaces develop more complex features.

With access to Fermi surfaces of (effectively) mixed surface MXenes, we can next estimate their properties. Since MXenes are known to exhibit excellent electrical conductivity, we evaluated the sheet conductivity within the constant relaxation time approximation (in $x$ direction) as

$$
\begin{aligned}
\sigma_{2 \mathrm{D}} & =\frac{e^{2}}{2 \pi^{2} \hbar} \tau_{0} \int_{E=E_{F}} \frac{v_{x}^{2}(k)}{|v(k)|} d k_{F} \\
& =\frac{e^{2}}{2 \pi^{2} \hbar} \tau_{0} \frac{\left\langle v_{F}\right\rangle}{2} \cdot l_{F},
\end{aligned}
$$

see Supplemental Material for more detailed description [31].

The sheet conductivities are shown in Fig. 4(a). The solid lines show the results from the full integral [Eq. (1a)] and the dashed lines from average Fermi-velocity approximation [Eq. (1b)]. All four considered MXenes show similar $\sigma_{2 \mathrm{D}} / \tau_{0}$, mostly falling within $2-41 / \Omega \square \mathrm{s}$. $\mathrm{Ti}_{2} \mathrm{CO}_{2}$ is semiconductor and thus does not possess well-defined Fermi-velocity, and its conductivity is here taken to be zero. The approximation [Eq. (1b)] works very well and thus by inspecting the $\left\langle v_{F}\right\rangle$ and $l_{F}$ shown in Figs. 4(c), 4(d) we can study their roles in governing the conductivity. Fermi-surface lengths and average Fermi velocity are similar in $\mathrm{Ti}_{3} \mathrm{C}_{2}, \mathrm{Ti}_{2} \mathrm{C}$, and $\mathrm{Ti}_{2} \mathrm{~N}$. $\mathrm{Ti}_{4} \mathrm{~N}_{3}$ shows 

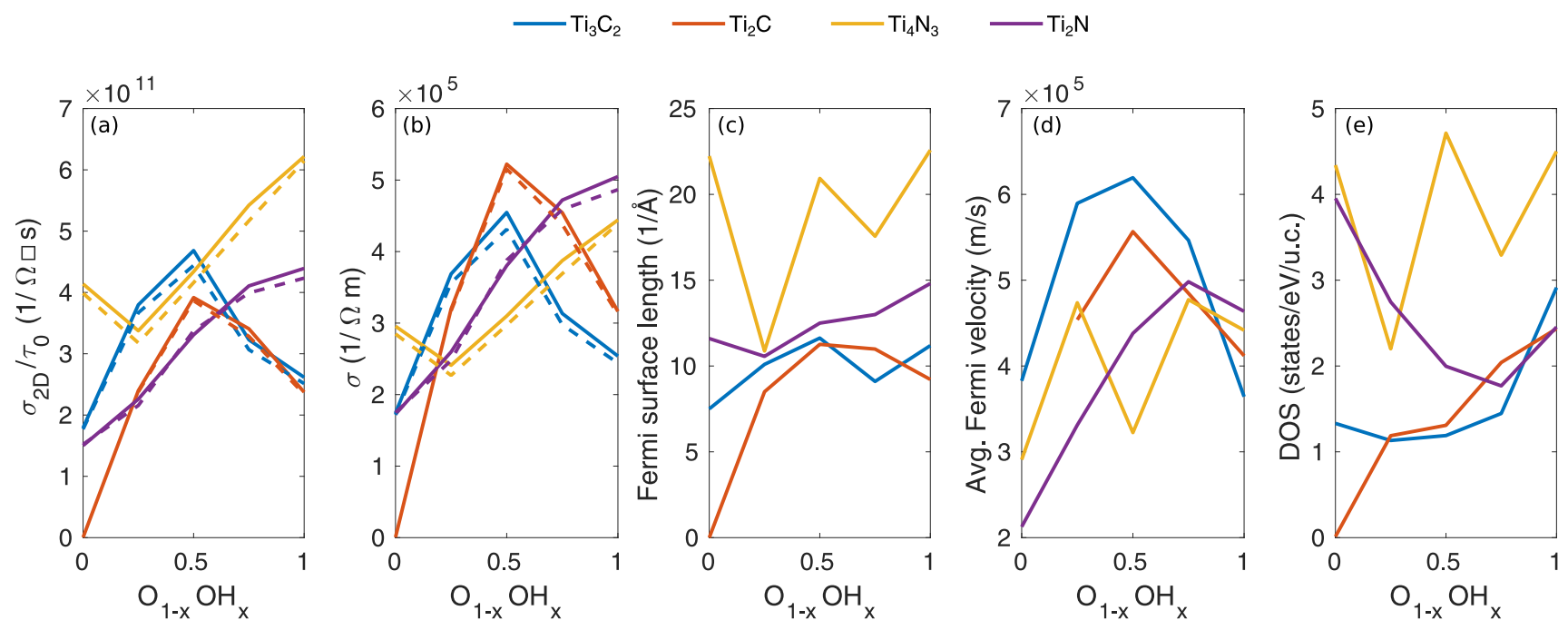

FIG. 4. Fermi-surface properties for all considered materials: (a) sheet conductivity, (b) conductivity, (c) Fermi-surface length, (d) Fermi velocity, and (e) density of states. The solid and dashed lines in (a) and (b) refer to sheet conductivity calculated from the full integral [Eq. (1a)] and from the average Fermi-velocity approximation [Eq. (1b)].

larger total length due to large number of states crossing the Fermi level, but the average Fermi velocities in these states are low. Overall, our results indicate that by simply tuning the surface composition the sheet conductivity cannot be changed by more than about a factor of two.

Trends in electrical conductivity are often evaluated from the density of states (DOS) at Fermi level, since $l_{F} \sim$ $D_{2 \mathrm{D}}\left(E_{F}\right)\left\langle v_{F}\right\rangle$ (cf. Supplemental Material [31]). The DOS at Fermi level are presented in Fig. 4(e) and shows that the trends in the conductivity changes with composition or between different materials cannot be extracted solely from the DOS due to missing the effect of $\left\langle v_{F}\right\rangle$.

Experimentally measuring the sheet conductivity of MXene monolayers is challenging, but the conductivity of MXene films has been reported in several publications. In the case of $\mathrm{Ti}_{3} \mathrm{C}_{2}$ films, conductivities above $10^{5} \mathrm{~S} / \mathrm{m}$ have been reported often [4,33-35]. Recently a value as high as $1.1 \times 10^{6}$ $\mathrm{S} / \mathrm{m}$ was achieved for a high crystalline quality $\mathrm{Ti}_{3} \mathrm{C}_{2}$ monolayer [36]. For other considered materials, the reports are more scarce, but nevertheless fairly similar values can be found: $5 \times 10^{5} \mathrm{~S} / \mathrm{m}$ for $\mathrm{Ti}_{2} \mathrm{~N}$ [37], and $2.3 \times 10^{5} \mathrm{~S} / \mathrm{m}$ for $\mathrm{Ti}_{2} \mathrm{C}$ [38]. It is worth noting, that the conductivity may be governed by interflake contacts and thus also sensitive to the presence of any intercalated species [39].

In order to estimate the film conductivity, we calculate $\sigma=$ $\left(\sigma_{2 \mathrm{D}} / \tau_{0}\right) \cdot \tau_{0} / t$, where $\tau_{0}$ is the relaxation time and $t$ is the interlayer separation taken from experiments: 10.3, 7.5 [1], 14 [40], and $8.7 \AA$ [37] for $\mathrm{Ti}_{3} \mathrm{C}_{2}, \mathrm{Ti}_{2} \mathrm{C}, \mathrm{Ti}_{4} \mathrm{~N}_{3}$, and $\mathrm{Ti}_{2} \mathrm{~N}$, respectively. Note, that the interlayer separation depends on the preparation method, as, e.g., intercalation of $\mathrm{Li}$ can increase the spacing by a few $\AA$. Here we do not attempt to evaluate $\tau_{0}$ from first-principles calculations, but instead from the comparison to experimentally measured conductivities. In Fig. 4(b) we show the results for $\tau_{0}=1 \mathrm{fs}$, which yields $\sigma$ values comparable to majority of the experimental reports. The result of Ref. [36] can be reproduced by taking slightly larger $\tau \approx 2-3 \mathrm{fs}$, depending on the surface composition. The lower $\tau_{0}$ in earlier reports may then be ascribed to larger number of defects caused by the strong etching, although the interflake contacts may also contribute.

\section{Angle-resolved photoemission spectrum (ARPES)}

To verify that our calculations properly capture the main features in the electronic structures of MXenes, we compare them to the only available experimental piece of information: the ARPES results for $\mathrm{Ti}_{3} \mathrm{C}_{2}$ reported in Ref. [10]. The experimental data is reproduced in Fig. 5(d) and shows: (i) flat band at the Fermi level, (ii) band with negative dispersion starting at $-1.5 \mathrm{eV}$, (iii) band with negative dispersion starting at $-2 \mathrm{eV}$, (iv) flat band at $-3 \mathrm{eV}$, (v) flat band segment at $-4 \mathrm{eV}$, (vi) broad flat band between -5 and $-6 \mathrm{eV}$, and (vii) broad flat band between -8 and $-9 \mathrm{eV}$. The composition was determined to be $\mathrm{Ti}_{3} \mathrm{C}_{2} \mathrm{~F}_{0.8} \mathrm{O}_{0.8}$.

In Fig. 5(a) we replot the effective band structure of $\mathrm{Ti}_{3} \mathrm{C}_{2}\left(\mathrm{O}_{0.5} \mathrm{OH}_{0.5}\right)_{2}$ overlaid with the pseudohydrogenated band structure from Fig. 1(a), but in a wider energy range. As discussed, the nonbonding Ti bands at and above $0 \mathrm{eV}$ and the $\mathrm{Ti}-\mathrm{C}$ bonding bands between -4 and $0 \mathrm{eV}$ are well reproduced in the pseudohydrogenated systems. The Ti-O bands between -7 and $-4 \mathrm{eV}$ are not reproduced very well, and the $\mathrm{O}-\mathrm{H}$ states at $-9 \mathrm{eV}$ are understandably poorly described by pseudohydrogens. Also some parts of the effective band structure are more strongly smeared by the surface group disorder than others, whereas the pseudohydrogen band structures contain no disorder. These factors limit the use of the pseudohydrogenated structures for reproducing ARPES results of deeper states.

Since the features close to Fermi level [features (i)-(iii) above] are properly described by pseudohydrogen models, we can start by comparing them to the band structures in Fig. 2 . Feature (i) indicates that the nonbonding band at $\Gamma$ point must be occupied, and therefore $\mathrm{O}$ content must be less than about 0.25 . The gap of about $1.5 \mathrm{eV}$ between features (i) and (ii) would match to the pure $\mathrm{OH}$-terminated surface. We then calculated effective band structures for few different mixtures 

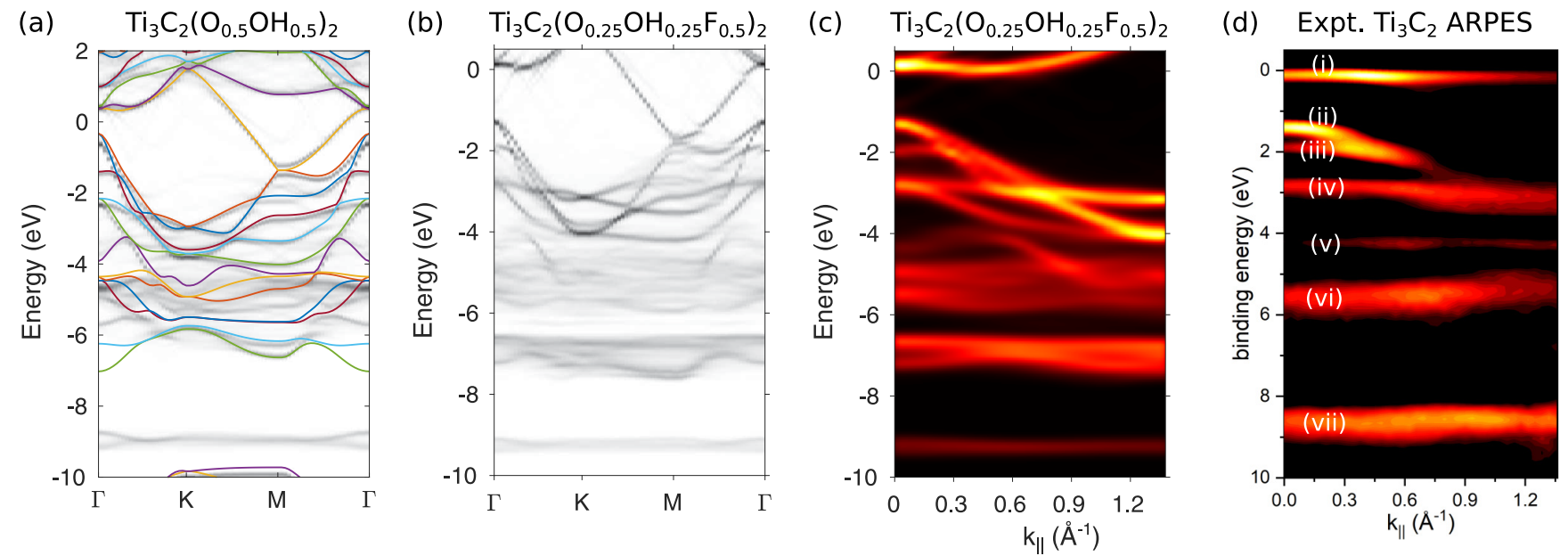

FIG. 5. (a) Calculated effective band structure of $\mathrm{Ti}_{3} \mathrm{C}_{2}\left(\mathrm{O}_{0.5} \mathrm{OH}_{0.5}\right)_{2}$ overlaid with the band structure from pseudohydrogenated system. (b) Effective band structure of $\mathrm{Ti}_{3} \mathrm{C}_{2}\left(\mathrm{O}_{0.25} \mathrm{OH}_{0.25} \mathrm{~F}_{0.5}\right)_{2}$. (c) The same as (b), but showing only the $\Gamma$-K line, broadened, and color map chosen to match that in the experiments. (d) Experimentally measured ARPES spectrum of $\mathrm{Ti}_{3} \mathrm{C}_{2} \mathrm{~T}_{x}$, adapted from Ref. [10].

with small $\mathrm{O}$ content and found overall best agreement with $\mathrm{Ti}_{3} \mathrm{C}_{2}\left(\mathrm{O}_{0.25} \mathrm{OH}_{0.25} \mathrm{~F}_{0.5}\right)_{2}$, as shown in Fig. 5(b) and processed such that it is more comparable to the experimental spectrum in Fig. 5(c). In this case, features (i)-(iv) are all reproduced very well. The EBS also shows flat band away from the $\Gamma$ point at about $-4 \mathrm{eV}$, matching well with feature (v) in the experimental spectrum. We also reproduce fairly well the broad band between -5 and $-6 \mathrm{eV}$, feature (vi). The lowest-energy feature (vii) is not reproduced in our calculations. The band at $-7 \mathrm{eV}$ in EBS originates from Ti-F bonds and the band at below $-9 \mathrm{eV}$ from $\mathrm{O}-\mathrm{H}$ bonds. Since semilocal functionals, such as PBEsol one used here, are known to underestimate band widths we carried out calculations for selected pure and pseudo-H surfaces using HSE06 functional and the results are shown in Fig. S3 [31]. While the main Ti-C and Ti-O related bands appear rather similar, HSE06 leads to larger gap opening at the $\Gamma$ point and deeper Ti-F band by $0.5-1 \mathrm{eV}$. Based on this and since experimentally measured sample should contain significant amount of $\mathrm{F}$, we ascribe feature (vii) to Ti-F bonds, in agreement with the discussion in Ref. [10]. A more detailed comparison would involve evaluation of the transition matrix elements and accounting for the many-body effects via, e.g., $G W$ calculations.

\section{CONCLUSIONS}

In conclusion, we presented a study of the fermiology of titanium carbide and nitride MXenes via density-functional theory calculations. We first calculated the effective band structures for surfaces with mixtures of $\mathrm{O}$ and $\mathrm{OH}$ groups. The electronic structure near the Fermi level was found to evolve gradually with the composition, and we further showed they are very well reproduced by surfaces fully terminated with pseudohydrogenated $\mathrm{OH}$ groups. With the pseudohydrogenated surfaces we could readily access Fermisurface properties, such as Fermi-surface area (or length in the case of 2D materials) and Fermi velocity. We calculated the electrical conductivity within the constant relaxation time approximation and by comparing to the experimentally determined conductivities we obtained relaxation time of about $1 \mathrm{fs}$. For the same relaxation time, the conductivities for all studied MXenes were rather similar and also fairly independent of the surface composition, changing at most by a factor of two. Finally, we compared our effective band structures to those obtained from recent ARPES experiments [10] for $\mathrm{Ti}_{3} \mathrm{C}_{2}$, where the best agreement was obtained using $\mathrm{Ti}_{3} \mathrm{C}_{2}\left(\mathrm{O}_{0.25} \mathrm{OH}_{0.25} \mathrm{~F}_{0.5}\right)_{2}$ model.

The approaches demonstrated here can be used also for other MXenes, including double MXenes [41] and even solid solutions in the $\mathrm{M}$ or $\mathrm{X}$ sublattice if their positions and those of the surface functional groups are uncorrelated. With the pseudohydrogenation approach it should be possible to calculate many other material properties of MXenes with an effective mixed surface composition, such as optical properties and electron-electron and electron-phonon scattering rates. It will also allow investigating how instabilities in electronic structure, leading to, e.g., magnetism or charge density waves, depend on the surface composition.

\section{ACKNOWLEDGMENTS}

We thank Prof. N. Koch and Dr. T. Schultz for providing us the original ARPES data, previously published in Ref. [10]. We are grateful to the Academy of Finland for support under Academy Research Fellow funding No. 311058. We also thank CSC-IT Center for Science Ltd. for generous grants of computer time.
[1] M. Naguib, O. Mashtalir, J. Carle, V. Presser, J. Lu, L. Hultman, Y. Gogotsi, and M. W. Barsoum, ACS Nano 6, 1322 (2012).

[2] Y. Gogotsi and B. Anasori, ACS Nano 13, 8491 (2019).
[3] M. Naguib, M. Kurtoglu, V. Presser, J. Lu, J. Niu, M. Heon, L. Hultman, Y. Gogotsi, and M. W. Barsoum, Adv. Mater. 23, 4248 (2011). 
[4] F. Shahzad, M. Alhabeb, C. B. Hatter, B. Anasori, S. Man Hong, C. M. Koo, and Y. Gogotsi, Science 353, 1137 (2016).

[5] B. Anasori, M. R. Lukatskaya, and Y. Gogotsi, Nature Rev. Mater. 2, 16098 (2017).

[6] Z. Fu, N. Wang, D. Legut, C. Si, Q. Zhang, S. Du, T. C. Germann, J. S. Francisco, and R. Zhang, Chem. Rev. 119, 11980 (2019).

[7] E. Lee and D.-J. Kim, J. Electrochem. Soc. 167, 037515 (2020).

[8] I. Matsuda and S. Hasegawa, J. Phys.: Condens. Matter 19, 355007 (2007).

[9] K. Lasek, J. Li, S. Kolekar, P. M. Coelho, L. Guo, M. Zhang, Z. Wang, and M. Batzill, Surf. Sci. Rep. 76, 100523 (2021).

[10] T. Schultz, N. C. Frey, K. Hantanasirisakul, S. Park, S. J. May, V. B. Shenoy, Y. Gogotsi, and N. Koch, Chem. Mater. 31, 6590 (2019).

[11] L. Chen, P. Cheng, and K. Wu, J. Phys.: Condens. Matter 29, 103001 (2017).

[12] T. Ouisse and M. W. Barsoum, Mater. Res. Lett. 5, 365 (2017).

[13] Y. Xie and P. R. C. Kent, Phys. Rev. B 87, 235441 (2013).

[14] M. Khazaei, M. Arai, T. Sasaki, C.-Y. Chung, N. S. Venkataramanan, M. Estili, Y. Sakka, and Y. Kawazoe, Adv. Funct. Mater. 23, 2185 (2013).

[15] M. Khazaei, A. Ranjbar, M. Arai, T. Sasaki, and S. Yunoki, J. Mater. Chem. C 5, 2488 (2017).

[16] T. Hu, H. Zhang, J. Wang, Z. Li, M. Hu, J. Tan, P. Hou, F. Li, and X. Wang, Sci. Rep. 5, 16329 (2015).

[17] R. Ibragimova, M. J. Puska, and H.-P. Komsa, ACS Nano 13, 9171 (2019).

[18] R. Ibragimova, P. Erhart, P. Rinke, and H.-P. Komsa, J. Phys. Chem. Lett. 12, 2377 (2021).

[19] T. B. Boykin and G. Klimeck, Phys. Rev. B 71, 115215 (2005).

[20] T. B. Boykin, N. Kharche, G. Klimeck, and M. Korkusinski, J. Phys.: Condens. Matter 19, 036203 (2007).

[21] W. Ku, T. Berlijn, and C.-C. Lee, Phys. Rev. Lett. 104, 216401 (2010).

[22] V. Popescu and A. Zunger, Phys. Rev. Lett. 104, 236403 (2010).

[23] G. Kresse and J. Furthmüller, Comput. Mater. Sci. 6, 15 (1996).

[24] G. Kresse and J. Furthmüller, Phys. Rev. B 54, 11169 (1996).

[25] P. E. Blöchl, Phys. Rev. B 50, 17953 (1994).
[26] J. P. Perdew, A. Ruzsinszky, G. I. Csonka, O. A. Vydrov, G. E. Scuseria, L. A. Constantin, X. Zhou, and K. Burke, Phys. Rev. Lett. 100, 136406 (2008).

[27] P. V. C. Medeiros, S. Stafström, and J. Björk, Phys. Rev. B 89, 041407(R) (2014).

[28] G. K. Madsen, J. Carrete, and M. J. Verstraete, Comput. Phys. Commun. 231, 140 (2018).

[29] A. M. Ganose, A. Searle, A. Jain, and S. M. Griffin, J. Open Source Software 6, 3089 (2021).

[30] T. Hu, Z. Li, M. Hu, J. Wang, Q. Hu, Q. Li, and X. Wang, J. Phys. Chem. C 121, 19254 (2017).

[31] See Supplemental Material at http://link.aps.org/supplemental/ 10.1103/PhysRevB.104.035408 for results and definitions of $3 \mathrm{D}$ quantities and their relation to the $2 \mathrm{D}$ quantities discussed in the main text, and the effects of functional group arrangement, layer charging, and hybrid functional on the band structures.

[32] J. Häglund, A. Fernández Guillermet, G. Grimvall, and M. Körling, Phys. Rev. B 48, 11685 (1993).

[33] J. Halim, M. R. Lukatskaya, K. M. Cook, J. Lu, C. R. Smith, L.-Å. Näslund, S. J. May, L. Hultman, Y. Gogotsi, P. Eklund et al., Chem. Mater. 26, 2374 (2014).

[34] Z. Ling, C. E. Ren, M.-Q. Zhao, J. Yang, J. M. Giammarco, J. Qiu, M. W. Barsoum, and Y. Gogotsi, Proc. Natl. Acad. Sci. 111, 16676 (2014).

[35] J. Liu, Z. Liu, H.-B. Zhang, W. Chen, Z. Zhao, Q.-W. Wang, and Z.-Z. Yu, Adv. Electron. Mater. 6, 1901094 (2020).

[36] A. Lipatov, A. Goad, M. J. Loes, N. S. Vorobeva, J. Abourahma, Y. Gogotsi, and A. Sinitskii, Matter 4, 1413 (2021).

[37] B. Soundiraraju and B. K. George, ACS Nano 11, 8892 (2017).

[38] J. Halim, I. Persson, E. J. Moon, P. Kühne, V. Darakchieva, P. O. Å. Persson, P. Eklund, J. Rosen, and M. W. Barsoum, J. Phys.: Condens. Matter 31, 165301 (2019).

[39] J. L. Hart, K. Hantanasirisakul, A. C. Lang, B. Anasori, D. Pinto, Y. Pivak, J. T. van Omme, S. J. May, Y. Gogotsi, and M. L. Taheri, Nature Commun. 10, 522 (2019).

[40] P. Urbankowski, B. Anasori, T. Makaryan, D. Er, S. Kota, P. L. Walsh, M. Zhao, V. B. Shenoy, M. W. Barsoum, and Y. Gogotsi, Nanoscale 8, 11385 (2016).

[41] B. Anasori, Y. Xie, M. Beidaghi, J. Lu, B. C. Hosler, L. Hultman, P. R. C. Kent, Y. Gogotsi, and M. W. Barsoum, ACS Nano 9, 9507 (2015). 